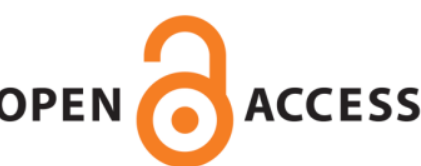

OPEN ACCESS

UWS Academic Portal

\title{
Estimation of minimum alerting boundaries in winds for small unmanned aircraft systems
}

Fang, Scott Xiang; O'Young, Siu; Rolland, Luc

Published in:

Journal of Aerospace Information Systems

DOI:

$10.2514 / 1.1010619$

E-pub ahead of print: 17/07/2018

Document Version

Peer reviewed version

Link to publication on the UWS Academic Portal

Citation for published version (APA):

Fang, S. X., O'Young, S., \& Rolland, L. (2018). Estimation of minimum alerting boundaries in winds for small unmanned aircraft systems. Journal of Aerospace Information Systems, 15(9), 530-543.

https://doi.org/10.2514/1.1010619

\section{General rights}

Copyright and moral rights for the publications made accessible in the UWS Academic Portal are retained by the authors and/or other copyright owners and it is a condition of accessing publications that users recognise and abide by the legal requirements associated with these rights.

Take down policy

If you believe that this document breaches copyright please contact pure@uws.ac.uk providing details, and we will remove access to the work immediately and investigate your claim. 


\title{
Estimation of Minimum Alerting Boundaries in Winds for Small UAS Detect-and-Avoid Systems
}

\author{
Scott Xiang Fang* and Siu O'Young ${ }^{\dagger}$ \\ Faculty of Engineering and Applied Science, Memorial University, St. John's, NL, AlB 3X5, Canada \\ Luc Rolland \\ School of Engineering and Computing, University of West Scotland, Paisley, Scotland, PA1 2BE, UK
}

\begin{abstract}
To achieve a level of safety equivalent to manned aircraft in the national airspace system, the pilot-in-command of small unmanned aircraft systems should be able to maintain situational awareness and make necessary maneuvres to avoid potential conflicts with nearby air traffic during mid-air encounters. However, due to ever-changing flight environments, the pilot-incommand often mis-estimates conflict risks and cannot engage the appropriate maneuvers to prevent mid-air traffic from violating the safety boundaries of small unmanned aircraft systems. To fix this problem, a Monte Carlo simulation-based method is therefore introduced and designed in this paper to quantitatively determine the alerting threshold boundaries in various wind conditions for detect-and-avoid systems to help the pilot-in-command identify hazards and trigger avoidance maneuvers before a safety boundary violation occurs.
\end{abstract}

\section{Nomenclature}

$\beta=$ detected traffic aircraft bearing angle

$d_{x} \quad=\quad$ traffic aircraft relative position on the $x$ axis (latitude)

$d_{y}=$ traffic aircraft relative position on the $y$ axis (longitude)

$d_{h} \quad=\quad$ traffic aircraft relative position on the $h$ axis (altitude)

m $=$ aircraft horizontal maneuvers

$\psi \quad=$ aircraft heading angle

$r \quad=$ horizontal range

$\dot{r} \quad=$ horizontal range rate

$\tau_{\text {mod }}=$ modified range tau

$t_{\mathrm{cPA}} \quad=$ time to the horizontal closest point of approach

$v_{r x} \quad=$ horizontal relative velocity on the $x$ axis (latitude)

*PhD Candidate, Faculty of Engineering and Applied Science, Memorial University, St. John's, NL, Canada, A1B 3X5.

${ }^{\dagger}$ Professor, Faculty of Engineering and Applied Science, Memorial University, St. John's, NL, A1B 3X5, Canada.

${ }^{\ddagger}$ Lecturer, School of Engineering and Computing, University of West Scotland, Paisley, Scotland, PA1 2BE, UK. 


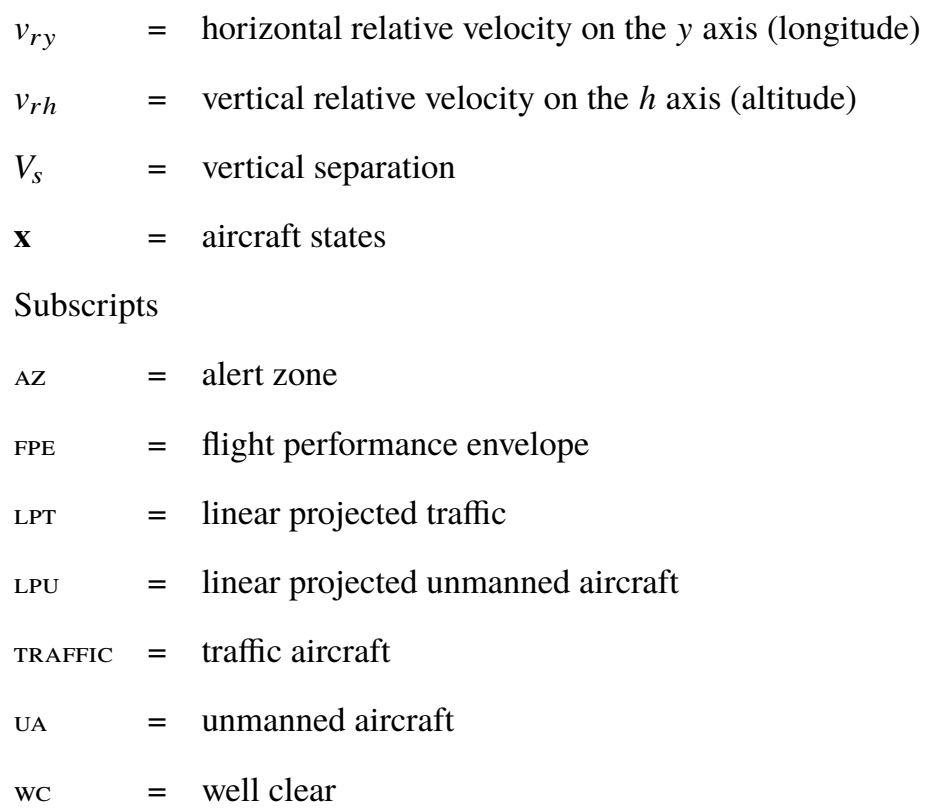

\section{Introduction}

$\mathrm{T}$ HE ability to Detect and Avoid (DAA) other aircraft during flight operations is an essential part of regulations governing the general operation of aircraft in the National Airspace System (NAS). On manned aircraft, human pilots are trained to communicate with and follow instructions from Air Traffic Control (ATC) on the ground, check equipment on the aircraft, and look through the windows of the aircraft to detect collision threats and maneuver to avoid other aircraft [1]. However, because Unmanned Aircraft Systems (UAS) currently lack this ability, the Federal Aviation Administration (FAA) has not allowed UAS to be integrated into the NAS with manned aircraft. All UAS operations can therefore only be carried out in limited operational areas in the NAS under Certificates of Waiver or Authorization (COA). Nevertheless, as industry pushes for the increased use of more UAS, the FAA has committed to modifying the current regulations. To help the industry gain operational experience and encourage researchers and system developers to solve the problems along the road to certify DAA systems, aviation authorities have proposed two near-term DAA solutions for integrating UAS into the NAS [2]:

1) Large UAS are required to have installed the same sensors as manned aircraft like airborne radar, Traffic Collision Avoidance System (TCAS), and Automatic Dependent Surveillance-Broadcast (ADS-B) to provide an equivalent level of safety as manned General Aviation (GA) aircraft [3]. In accord with the current regulations [4, 5], once these sensors are equipped, processed sensor information is transmitted from the Unmanned Aircraft (UA) to the Ground Control Station (GCS), where the Pilot-in-Command (PIC) analyzes the guidance from the DAA systems and the data from different sensors, and makes final decisions on whether maneuvers are needed to avoid other surrounding traffic. 
2) Small UAS are required to have installed newly-developed small, light, and low-power versions of airborne sensors or portable ground-based sensors for safe operations due to their limitations on Size, Weight, and Power (SWaP). Once equipped, processed sensor information is transmitted from the UA to the GCS, where the PIC analyzes the guidance from the DAA systems and the data from different sensors, and makes final decisions on whether maneuvers are needed to avoid other surrounding traffic.

\section{A. Problem Discussion}

The first solution for large UAS, discussed in Section [1 is a straightforward starting point for integrating UAS into the NAS with other manned GA aircraft in the near future, as those DAA systems have already been proven and certified on manned aircraft. The remaining challenges are therefore to modify the current regulations and operational procedures in the NAS, and to have additional training courses for air traffic controllers and pilots of large UAS and manned aircraft.

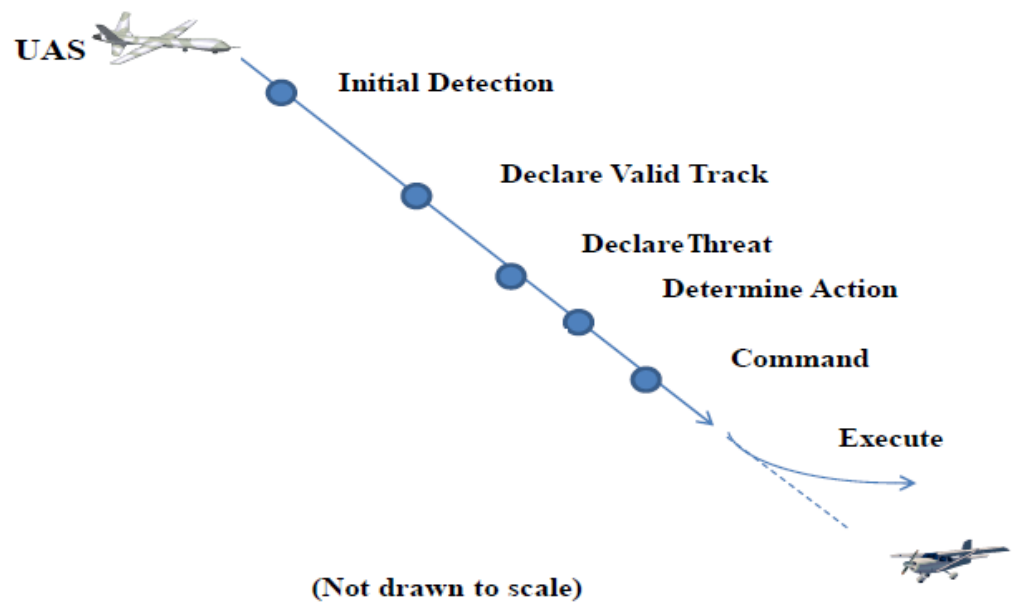

Fig. 1 DAA timeline diagram [6].

However, implementing the second near-term DAA solution for small UAS will be much harder for researchers and engineers in the UAS industry than implementing the first solution for large UAS, since there are no certified DAA systems that would allow small UAS to achieve the equivalent level of safety as manned GA aircraft. In addition, small UAS aerodynamic characteristics are very different from manned aircraft. The collision avoidance solutions for manned aircraft will not be suitable for small UAS. Generally, small UAS are light-weight (less than $25 \mathrm{~kg}$ ) and operate at a slow cruise airspeed (less than $100 \mathrm{kt}$ ) [7]; thus, the turning trajectories of small UAS and the time needed to complete turns are greatly affected by the wind conditions (i.e., wind speed and wind direction) during a level maneuver, whereas manned aircraft operate faster and are less affected by the wind during a turn. To reflect this reality, new specific collision avoidance solutions should be designed and implemented in the new DAA systems for small UAS to help the PIC quantitatively identify traffic hazards, assess collision risks and make appropriate maneuvers to avoid potential 
collisions, as depicted in Fig. 1 [6].

\section{B. Research Objective}

Following the DAA timeline in Fig. 11, the RAVEN project/has proposed a DAA system to help small UAS establish the DAA ability for future safe operations in the NAS, as described in Fig. 2 An ADS-B-based light-weight airborne sensor and a portable ground radar are employed in the Detect System for small UAS to detect and track cooperative and non-cooperative traffic nearby small UAS operating areas. Three quantifiable analysis modules are also developed in the Avoid System to carry out risk-quantitative analysis and decision-making for the PIC to improve situational awareness and to steer small UAS to safe regions (when required) during mid-air encounters [8].

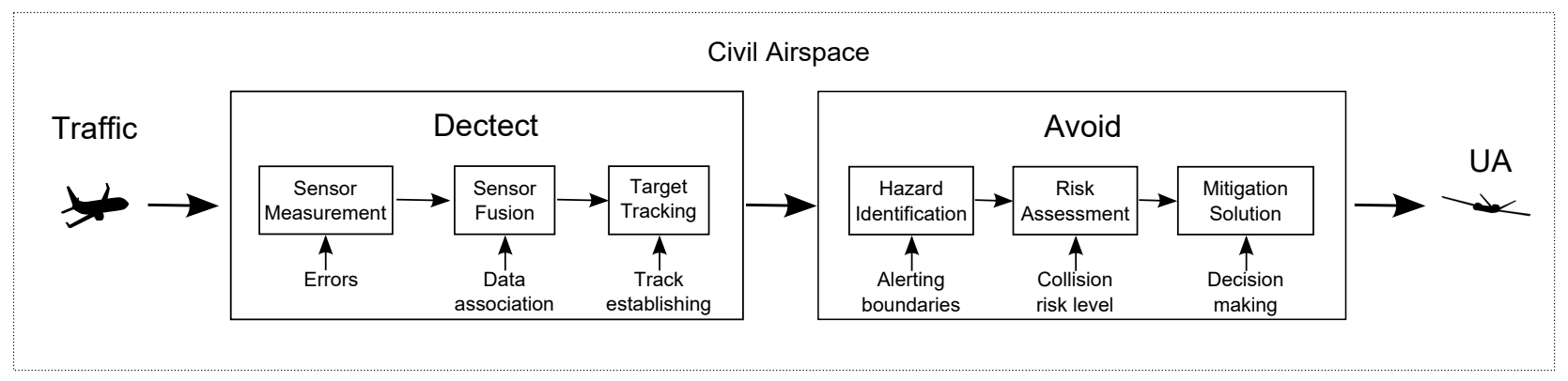

Fig. 2 Block diagram for the proposed DAA system.

The research described focuses on the hazard identification module (the first module in the Avoid System for the proposed DAA system), which is designed to help the PIC quantitatively estimate the alerting boundaries around small UA, and determine whether an avoidance maneuver is required to avoid a potential collision for small UAS. As shown in Fig. 3a the RTCA Special Committee 228 (SC-228) has determined two layers of critical UAS safety boundaries around the UA to quantitatively predict and resolve a potential conflict in a mid-air encounter: the Near Mid-Air Collision (MNAC) boundary and the Well Clear (WC) boundary [2].

- The NMAC boundary is a standard cylinder: $500 \mathrm{ft}$ in radius horizontally, and $\pm 100 \mathrm{ft}$ in height vertically.

- The WC boundary is not only a spatial separation in range, but also a temporal separation in time [9, 10]. For example, during a head-on encounter, the radius of the WC cylinder in the front of the UA is a 35-second travel distance between the intruder and the UA, and the radius of the WC cylinder in the back of the UA is $4000 \mathrm{ft}$. In addition, the height of the WC cylinder is $\pm 450 \mathrm{ft}$ from the UA.

As shown in Fig. 3b to prevent violating two critical safety boundaries (WC and NMAC) in a mid-air encounter, we further introduce two new alerting threshold boundaries (outside of the WC boundary and the NMAC boundary, respectively):

*The RAVEN project is based at Memorial University of Newfoundland, Canada, and their main research objective is to develop intelligent DAA systems to allow small UAS to share the same airspace with manned aviation. 
- A Well Clear Alerting Threshold (WCAT) boundary is located at an outer layer outside of the WC cylinder. It is defined as the closest range boundary at which the UA can still remain WC with the traffic by a horizontal maneuver within the Flight Performance Envelope (FPE).

- A Collision Avoidance Alerting Threshold (CAAT) boundary is located at an outer layer outside of the NMAC cylinder. It is defined as the closest range boundary at which the UA can still avoid NMAC with the traffic by a horizontal maneuver within the FPE.

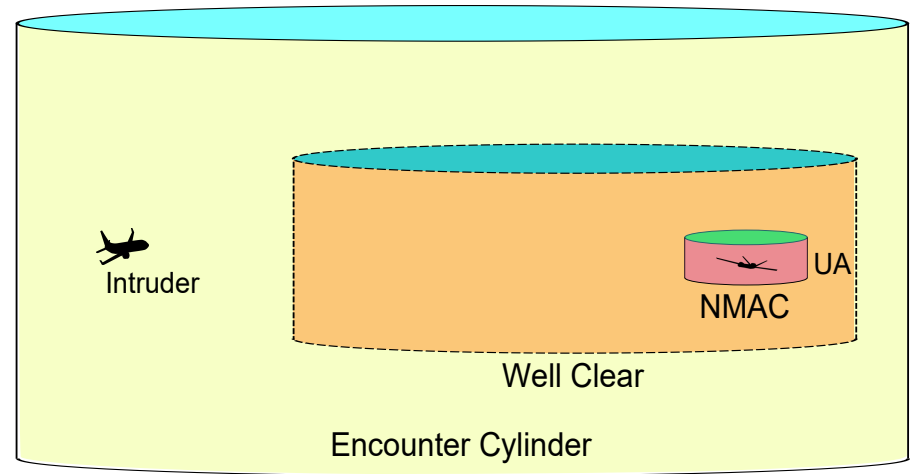

(a) Encounter cylinders.

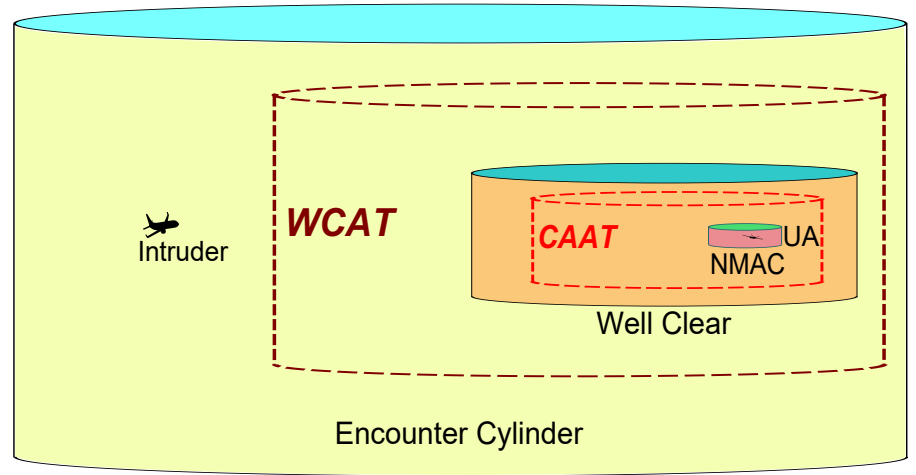

(b) Alerting threshold boundaries.

Fig. 3 Layered encounter model of the airspace nearby the UA.

On large UAS, these two alerting threshold boundaries (WCAT and CAAT) can be estimated through a set of mathematical equations derived in [11]; however, on small UAS, these two alerting threshold boundaries cannot be directly calculated, as small UAS are sensitive to ever-changing flight environments $Q$ as encounter geometries, relative velocities, and wind conditions (i.e., wind speed and wind direction). A Monte Carlo (MC) simulation method is therefore introduced and designed in this paper to handle these uncertainties; namely, the WCAT and CAAT boundaries are located by checking small UAS maneuvrabilities over all possible initial conditions of encounters and researched environments (i.e., different wind conditions, various relative velocities, and encounter geometries in all directions). Once completed, the analytical results from the MC simulations are used to form lookup tables to provide real-time WCAT and CAAT estimations for small UAS to issue appropriate maneuvering alerts during mid-air encounters, so that 
the PIC can be warned to trigger an avoidance maneuver at an appropriate time before a Loss of Well Clear (LoWC) or NMAC occurs. An outline of the paper is as follows: In Section III the formal definitions of alerting boundaries for mid-air encounters are given along with the related DAA parameters and terminologies. In Section III, two algorithms to determine small UAS WCAT and CAAT boundaries in winds are provided. In Section IV, the results from two MC simulations are discussed, analyzing the impact of WCAT and CAAT boundaries on wind uncertainties. Finally, conclusions and future work are presented in Section $\mathrm{V}$

\section{DAA Parameters and Terminologies}

To explain the MC method designed and carry out further quantitative analysis in hazard identification during mid-air encounters, it is necessary to identify the basic DAA parameters and terminologies that are related to, and will be used in, the research.

\section{A. DAA Parameters}

As shown in Fig. 4 , for convenience in the analysis and implementation, air traffic positions in mid-air encounters are defined on the local three-dimensional Cartesian coordinates $(x, y, h)$ with respect to the UA, on which the $x$ axis is pointing to the north for the latitude position, the $y$ axis is pointing to the east for the longitude position, and the $h$ axis is pointing up for the altitude position. In addition, $\psi$ is the traffic heading relative to the north, and $v$ is the horizontal velocity of the traffic in a given three-dimensional space.

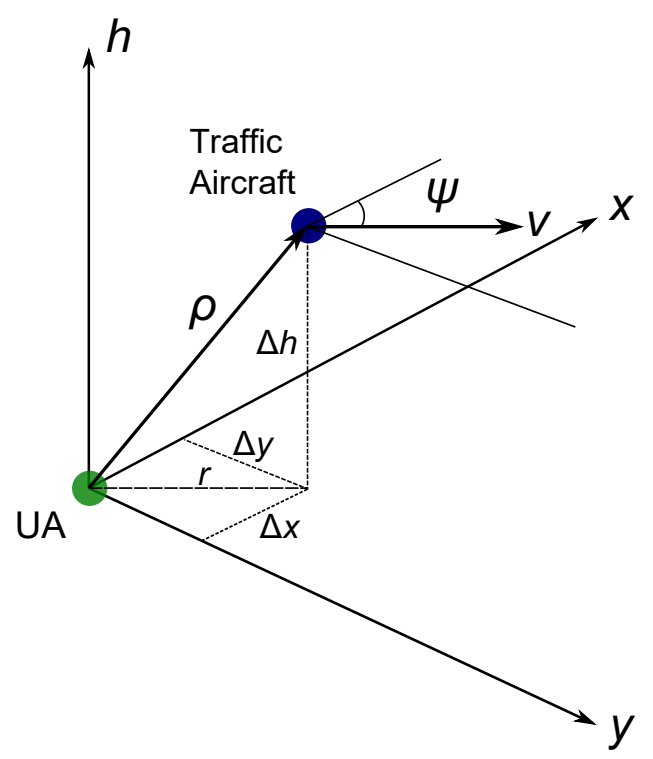

Fig. 4 Aircraft positions in a given three-dimensional space [12]. 
In Fig. 4 ( $(\Delta x, \Delta y, \Delta h)$ are local positions for the traffic relative to the UA. Assume that

$$
\begin{aligned}
& d_{x}=\Delta x=x_{\text {тRAFFC }}-x_{\mathrm{UA}} \\
& d_{y}=\Delta y=y_{\text {TRAFFC }}-y_{\mathrm{UA}} \\
& d_{h}=\Delta h=h_{\text {TRA } A \mathrm{AC}}-h_{\mathrm{UA}}
\end{aligned}
$$

The traffic velocities relative to the UA in the three-dimensional space are

$$
\begin{gathered}
v_{r x}=v_{\text {TRAFFC }} \cos \left(\psi_{\text {TRAFFC }}\right)-v_{\mathrm{UA}} \cos \left(\psi_{\mathrm{UA}}\right) \\
v_{r y}=v_{\text {TRAFHC }} \sin \left(\psi_{\mathrm{TRAFFIC}}\right)-v_{\mathrm{UA}} \sin \left(\psi_{\mathrm{UA}}\right) \\
v_{r h}=\dot{h}_{\text {TRAFTC }}-\dot{h}_{\mathrm{VAA}}
\end{gathered}
$$

Similarly, the horizontal range $r$ is

$$
r=\sqrt{d_{x}^{2}+d_{y}^{2}}
$$

The horizontal range rate $\dot{r}$ is

$$
\dot{r}=\frac{\mathrm{d} r}{\mathrm{~d} t}=\frac{d_{x} v_{r x}+d_{y} v_{r y}}{r}
$$

It is noted that the safe horizontal range to separate UA from other traffic often changes in mid-air encounters, in terms of various relative velocities between UA and traffic. Thus, to unify a safe separation threshold in risk assessment, a time-based range tau is introduced to evaluate the risk severity of potential collisions as [13]

$$
\tau=-\frac{r}{\dot{r}}(\text { if } \dot{r} \neq 0)
$$

Usually, a larger $\tau$ indicates lower collision risks and a smaller $\tau$ means higher collision risks. However, in the case of two tail-chase aircraft operated at similar velocities (i.e., range rate $\dot{r} \rightarrow 0$ ), the calculated range tau from Eq. 9 will stay high even when the intruder is in the NMAC cylinder (i.e., $r<500 \mathrm{ft}$ ). In addition, this range tau will not be able to provide sufficient alerting time to avoid a LoWC or even a NMAC when a traffic suddenly accelerates. Thus, another modified range tau, Distance MODification (DMOD) is used to provide a minimum range, at which to alert regardless of the range tau. This modified range tau $\left(\tau_{\text {mod }}\right)$ is defined as [13, 14]

$$
\tau_{\text {mod }}=-\frac{r-\left(D M O D^{2} / r\right)}{\dot{r}}=-\frac{r^{2}-D M O D^{2}}{r \dot{r}}(\text { if } r \dot{r} \neq 0)
$$

\footnotetext{
†DMOD: an absolute distance threshold to alert the collision risk in mid-air encounters, e.g., DMOD $=4000 \mathrm{ft}$ to alert the LoWC.
} 
where: $\mathrm{DMOD}=4000 \mathrm{ft}$ for the minimum of WC boundaries, and $\left(\mathrm{DMOD}^{2} / r\right)$ is a range buffer depending on the ratio of the DMOD and the horizontal range, i.e., $\left(\mathrm{DMOD}^{2} / r\right)=\mathrm{DMOD} \times\left(\frac{\mathrm{DMOD}}{r}\right)$.

Another important encounter parameter is the Horizontal Miss Distance (HMD) between the UA and other traffic in an encounter, which refers to the horizontal distance at the horizontal Closest Point of Approach (CPA) between two aircraft throughout the entire encounter [2, 15]. In general, the horizontal range of two aircraft is defined as the time-domain function

$$
r(t)=\sqrt{\left(x_{0}+v_{r x} t\right)^{2}+\left(y_{0}+v_{r y} t\right)^{2}}
$$

where $x_{0}$ and $y_{0}$ are the initial positions of the other traffic relative to the UA.

The minimal distance occurs when $\frac{\mathrm{d}\left(r(t)^{2}\right)}{\mathrm{d} t}=0$, so the time to CPA can be obtained by

$$
t_{\mathrm{cPA}}=\max \left(0,-\frac{x_{0} v_{r x}+y_{0} v_{r y}}{v_{r x}{ }^{2}+v_{r y}{ }^{2}}\right)
$$

where a positive $t_{\mathrm{cPA}}$ expresses closing geometries and zero indicates other non-closing geometries.

According to Eq. 11 and Eq. 12, the HMD of two aircraft in an encounter can be predicted from its current positions as [2]

$$
H M D=\sqrt{\left(d_{x}+v_{r x} t_{\mathrm{CPA}}\right)^{2}+\left(d_{y}+v_{r y} t_{\mathrm{cPA}}\right)^{2}}
$$

Similarly, the Vertical Miss Distance (VMD) between the UA and other traffic at the horizontal CPA can be predicted by

$$
V M D=d_{h}+v_{r h} t_{\mathrm{CPA}}
$$

\section{B. Formal Definitions for Mid-Air Encounters}

In this subsection, the formal definitions for NMAC and WC cylinders are given as follows:

Definition 1 (NMAC) The NMAC boundary is a standard cylinder around the UA. $R_{\mathrm{NMAC}}=500$ feet in radius horizontally, and $h_{\mathrm{NMAC}}= \pm 100$ feet in height vertically [2].

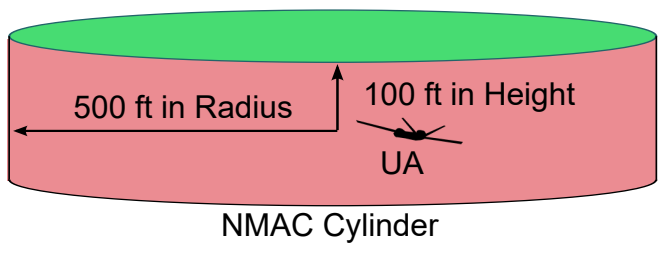

Fig. 5 NMAC cylinder diagram. 
Definition 2 (WC) For given: Modified Range Tau Threshold $\tau_{\text {mod }_{\mathrm{wc}}}^{*}=35 \mathrm{sec}$, Horizontal Miss Distance Threshold $H M D_{\mathrm{wc}}^{*}=D M O D_{\mathrm{wc}}=4000 \mathrm{ft}$, and Vertical Separation Threshold $V M D_{\mathrm{wc}}^{*}=450 \mathrm{ft}$, an intruder is within WC [2] when

$$
\left[0 \leq \tau_{m o d} \leq \tau_{m o d_{\mathrm{wc}}}^{*}\right]\left[H M D \leq H M D_{\mathrm{wc}}^{*}\right]\left[V_{s} \leq V M D_{\mathrm{wc}}^{*}\right]
$$

where: $\tau_{\text {mod }}$ is the modified range tau defined in Eq. 10, HMD is the horizontal miss distance at CPA defined in Eq. 13 , and $V_{s}$ is the vertical separation that can be calculated by $V_{s}=a b s\left(d_{h}\right)$ with Eq. 3

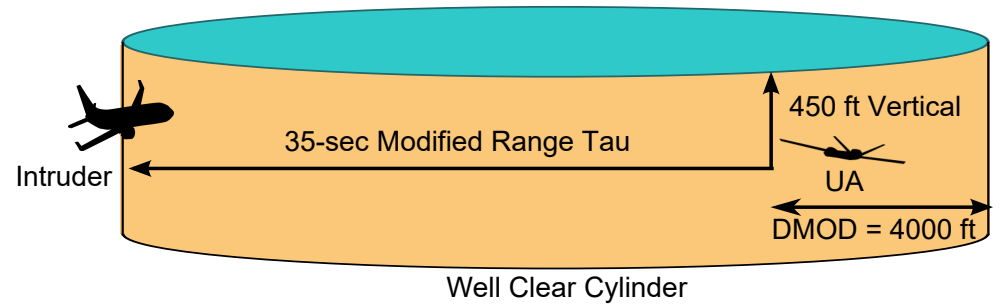

Fig. 6 WC cylinder diagram.

Assume that $\mathbf{x}=[x, y, h, \psi, v]$ are aircraft states for latitude, longitude, altitude, heading, and horizontal velocity,

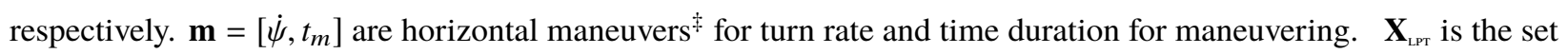
of all possible aircraft states for linear projected traffic (LPT). $\$ \mathbf{X}_{\mathrm{LPv}}$ is the set of all possible aircraft states for linear projected UA (LPU). $10 \mathbf{M}_{\mathrm{FPE}}^{\mathrm{wC}}$ is the set of all allowed UA horizontal maneuvers within the FPE that can be used for remaining WC, and $\mathbf{M}_{\mathrm{FPE}}^{\overline{\mathrm{MAC}}}$ is the same for UA to avoid NMACs.

Definition 3 (WCAT) For given: $\mathbf{x}_{\mathrm{TRAFFC}} \in \mathbf{X}_{\mathrm{LPT}}$ and $\mathbf{x}_{\mathrm{vA}} \in \mathbf{X}_{\mathrm{LP},}$, a traffic aircraft is outside of WCAT when

$$
\exists \mathbf{m}_{\mathrm{vA}} \in \mathbf{M}_{\mathrm{rPE}}^{\mathrm{wc}} \text { such that } \mathrm{WC}
$$

Definition 4 (CAAT) For given: $\mathbf{x}_{\text {тва丿ғт }} \in \mathbf{X}_{\mathrm{LPT}}$ and $\mathbf{x}_{\mathrm{LA}} \in \mathbf{X}_{\mathrm{Lv}}$, a traffic aircraft is outside of CAAT when

$$
\exists \mathbf{m}_{\mathrm{UA}} \in \mathbf{M}_{\mathrm{FPE}}^{\overline{\mathrm{MMC}}} \text { such that } \overline{\mathrm{NMAC}}
$$

\footnotetext{
$\$ \mathbf{m}$ refers to single-move horizontal maneuvers, e.g., a horizontal maneuver at $6 \%$ sec for 5 seconds.

$\S$ LPT refers to the traffic aircraft that are flying at constant velocities on straight line flights.

"I LPU refers to the UA that are flying at constant velocities on straight line flights.
} 


\section{Control Zones for Hazard Identification}

For hazard identification, three control zones around the UA are proposed and defined in Fig. 7 the hazard zone, the alert zone, and the safety zone. In the design, when traffic aircraft break into the hazard zone, the UA should take immediate maneuvers to avoid potential mid-air collisions; when traffic aircraft break into the alert zone, the UA may or may not respond to alerts from DAA systems, depending upon collision risk levels; and when traffic aircraft are outside of the alert zone, the UA are in the safety zone and can remain on the original flight course.

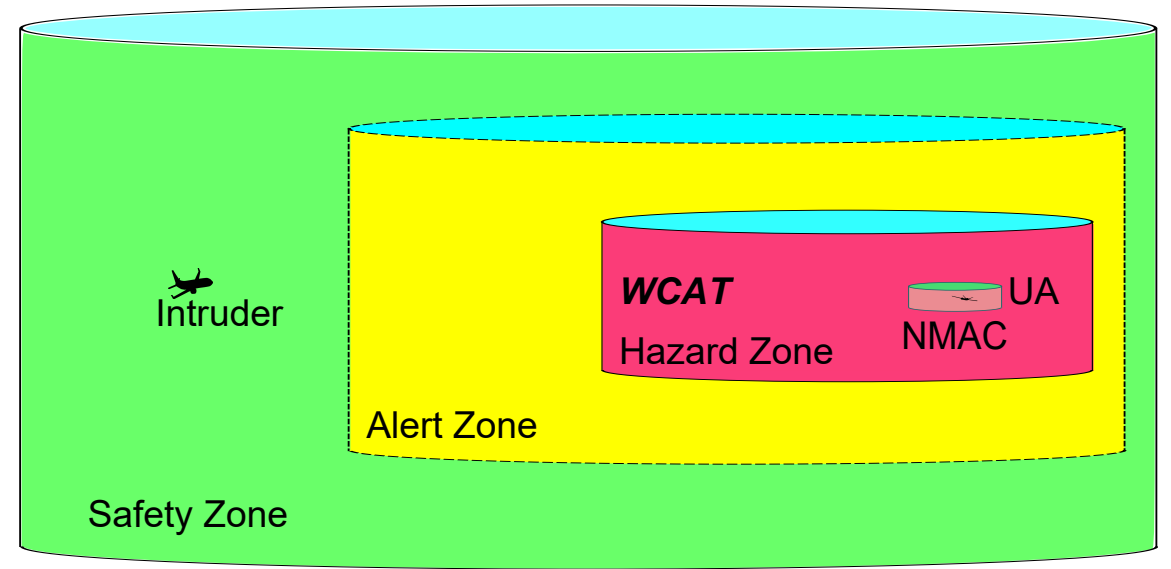

Fig. 7 Layered encounter zones of the UA.

As depicted in Fig. 7 the hazard zone in red refers to the WCAT of the UA. The safety zone in green refers to the areas outside the alert zone of the UA. The alert zone in yellow is a larger cylinder outside the WCAT, which is defined as follows [2]:

Definition 5 (Alert Zone) For given: Modified Range Tau Threshold $\tau_{\text {mod }_{\Lambda z}}^{*}=110 \mathrm{sec}$, Horizontal Miss Distance Threshold $H M D_{A z}^{*}=D M O D_{A z}=2 \mathrm{nmi}$, and Vertical Separation Threshold $V M D_{A z}^{*}=800 \mathrm{ft}$, an intruder is within the alert zone when

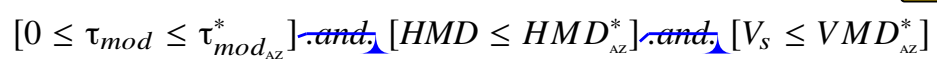

\section{Mitigation Categories in Mid-Air Encounters}

Two categories of UAS mitigation solutions are proposed to avoid LoWCs or NMACs in mid-air encounters:

- Self-separation refers to efforts carried out by the PIC with the guidance from DAA systems to avoid LoWCs when traffic aircraft are penetrating the alert zone of the UA with LoWCs projected.

- Collision avoidance refers to maneuvers triggered by DAA systems to avoid NMACs when traffic aircraft have entered the hazard zone and are close to breaching the CAAT boundary of the UA with NMACs projected. 


\section{Implementation for Hazard Identification}

To identify hazards for the UA during mid-air encounters, we should first locate boundaries for the alert zone and the hazard zone of the UA. As given in Section II] the alert zone of the UA can be easily calculated in real-time through Eq. 18, however, the hazard zone (i.e., the WCAT boundary) of the UA cannot be simply obtained through the statement in Definition 3 (WCAT) in real-time, because it usually requires a time-consuming Monte Carlo (MC) simulation to estimate the WCAT boundary for the UA in that encounter. Moreover, in the case of the breach of the hazard zone, we should locate the CAAT boundary for DAA systems to determine if collision avoidance maneuvers are required as a last resort to avoid potential NMACs. Similarly, the CAAT boundary cannot be calculated through the statement in Definition 4 (CAAT) in real-time, which also requires a time-consuming MC simulation to out the estimation.

To fix these problems, we develop two algorithms in this section, based on MC simulations, to locate WCAT and CAAT boundaries, and then establish WCAT and CAAT lookup tables for real-time hazard identification during UAS operations.

\section{A. Aircraft trajectory generation in the wind}

It is noted that small UA are usually light-weight (e.g., less than $25 \mathrm{~kg}$ ) and operate at a slow cruise airspeed (e.g., 75 kt), so their turning trajectories and the time needed to complete turns vary in different wind conditions (i.e., different wind speed and wind directions). As small UA turning trajectories look very different under various wind conditions in Fig. 8, wind effects have to be quantified in MC simulations to locate WCAT and CAAT boundaries for small UA.

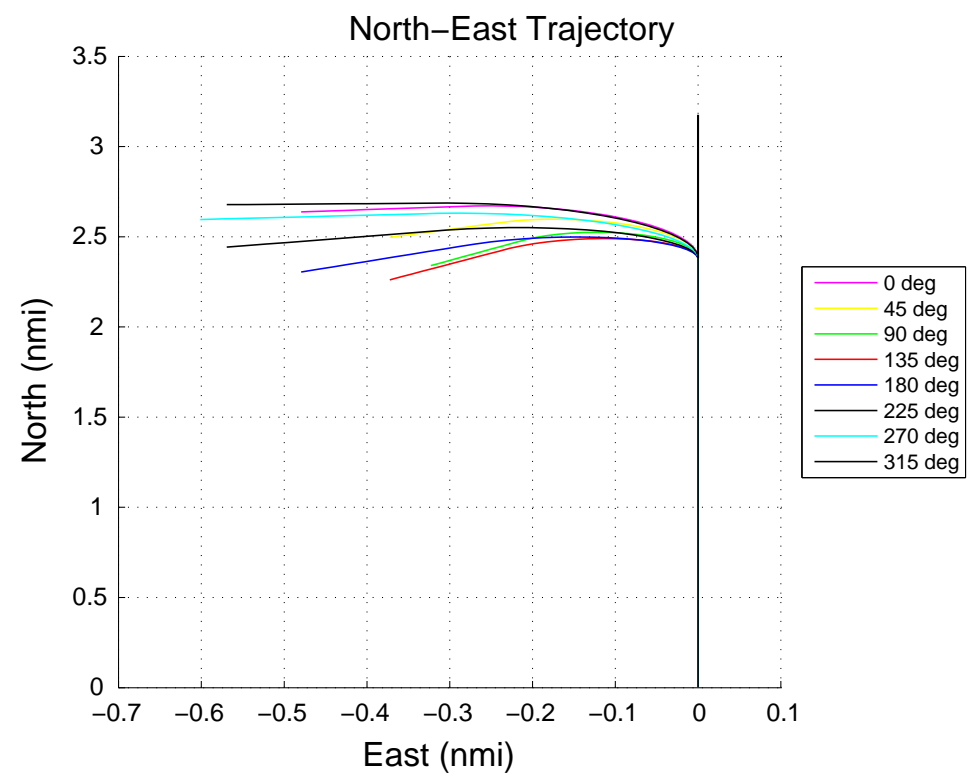

Fig. 8 Sample turning trajectories for a 20-second $2 g\left(6^{\circ} / \mathrm{sec}\right)$ left level turn $(75 \mathrm{kt} \mathrm{UA}$ under $20 \mathrm{kt}$ winds in various wind directions). 


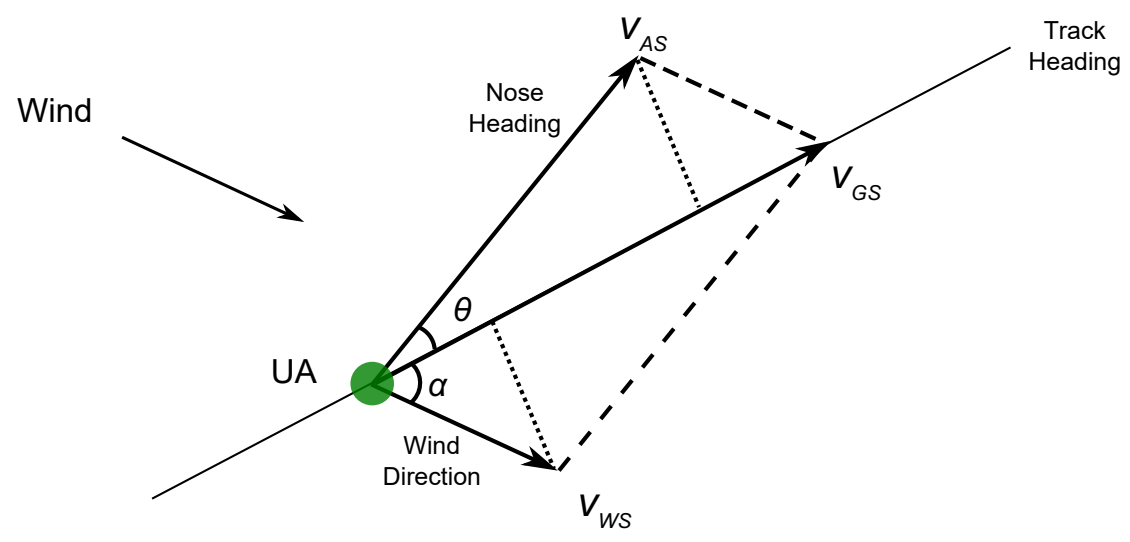

Fig. 9 Aircraft trajectory generation in the wind.

If we assume that the wind has only a horizontal component with wind speed $v_{\mathrm{ws}}$ and wind direction $\psi_{\text {wivo }}$, and that the UA is on the level flight with airspeed $v_{\mathrm{As}}$, ground speed $v_{\mathrm{cs}}$, and turn rate $\dot{\psi}$, then UA trajectories during level flights and horizontal maneuvers in the wind can be generated by the following equations, as depicted in Fig. 9 [16].

$$
\begin{aligned}
& \alpha(0)=\psi_{\text {WIND }}-\psi_{\text {тRRAK }}(0) \\
& \theta(0)=\arcsin \left(\frac{v_{\mathrm{ws}} \sin (\alpha(0))}{v_{\mathrm{As}}}\right) \\
& \psi_{\text {Nose }}(0)=\psi_{\text {TRACK }}(0)-\theta(0) \\
& \psi_{\text {NosE }}(n)=\psi_{\text {NoSE }}(n-1)+\dot{\psi}(n) \\
& v_{\mathrm{GS} x}(n)=v_{\mathrm{AS}} \cos \left(\psi_{\mathrm{NOSE}}(n)\right)+v_{\mathrm{wS}} \cos \left(\psi_{\mathrm{wIND}}\right) \\
& v_{\mathrm{cs} y}(n)=v_{\wedge \mathrm{s}} \sin \left(\psi_{\mathrm{NOSE}}(n)\right)+v_{\mathrm{ws}} \sin \left(\psi_{\mathrm{wND}}\right) \\
& x_{n}=x_{n-1}+v_{\mathrm{Gs}_{x}}(n) \\
& y_{n}=y_{n-1}+v_{\mathrm{cs} y}(n) \\
& h_{n}=h_{n-1}
\end{aligned}
$$

where: $n=[1,2,3, \ldots] \in \mathbb{N}^{+} . \theta$ is the angle between the UA nose heading $\psi_{\text {Nose }}$ and the UA ground track heading $\psi_{\text {тRаск }}$. $\alpha$ is the angle between the wind direction $\psi_{\text {шім }}$ and the UA ground track heading $\psi_{\text {тваск }}$. 


\section{B. Algorithms to determine WCAT and CAAT boundaries}

For simplicity, vertical boundaries of CAAT and WCAT are pre-defined at fixed heights; namely, the vertical boundary of CAAT is defined as $V M D_{\mathrm{wc}}^{*}=450 \mathrm{ft}$, and the vertical boundary of WCAT is defined as $V M D_{\mathrm{Az}}^{*}=800$ ft. Thus, only horizontal boundaries of WCAT and CAAT need to be determined via MC simulations by a series of co-altitude mid-air encounters.

To implement these MC simulations, as shown in Fig. 10, we introduce a web structure in the algorithm design, where traffic aircraft are initiated from inner circles to outer circles at bearing angles $\left[0^{\circ}, 360^{\circ}\right)$ with heading angles in $\left[0^{\circ}, 360^{\circ}\right)$; UA are initiated at the web center with the $0^{\circ}$ heading angle (to the north). At each bearing angle, we locate the shortest horizontal range between UA and traffic at which UA can avoid LoWCs or NMACs with traffic in all possible headings by one of the appropriate horizontal maneuvers.

In the implementation, two algorithms are developed for UA to determine WCAT and CAAT boundaries via MC simulations, i.e., Algorithm 1(Determine WCAT) and Algorithm2(Determine CAAT). For obtaining bounded WCAT and CAAT boundaries, as Definition 3 (WCAT) and Definition 4 (CAAT), two algorithms are designed with two assumptions: 1) traffic are on level flights with constant horizontal velocities throughout mid-air encounters, and 2) UA are on level flights or level maneuvers to avoid LoWCs or NMACs. Hence, the states of UA and traffic can be reduced to $[x, y, \psi, v, \dot{\psi}]$ for latitude, longitude, heading, horizontal velocity, and turn rate, respectively.

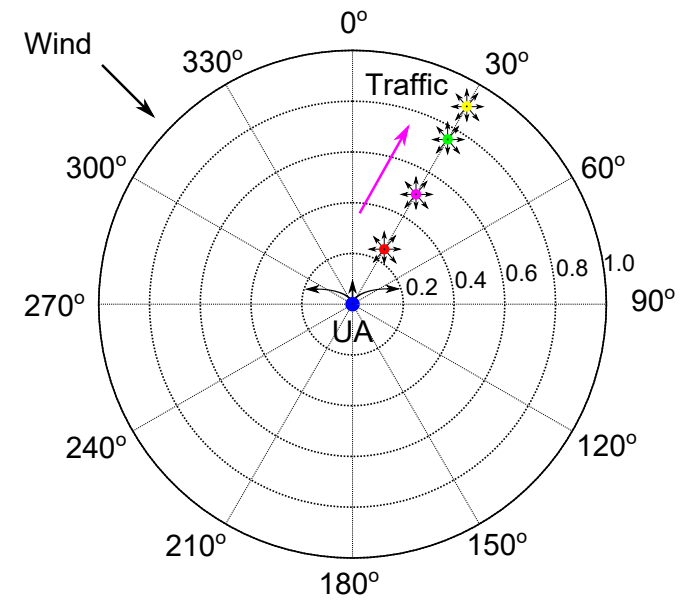

Fig. 10 A web structure for Monte Carlo simulations to determine WCAT and CAAT boundaries (bearing in deg and range in nmi).

The parameters and settings used in MC simulations to implement Algorithm 1 (Determine WCAT) and Algorithm 2 (Determine CAAT) are listed as follows:

- Based on the airspace in the low altitude below $5000 \mathrm{ft}$ at which small UA are most likely operated, assume that small UA are operated at airspeed $v_{\mathrm{UA}}=75 \mathrm{kt}$ and turn rate $\dot{\psi}_{\mathrm{UA}}=6 \% \mathrm{sec}$.

- Traffic are simulated at an airspeed range of $[50,300] \mathrm{kt}$ with an airspeed increment every $50 \mathrm{kt}$. 
- For WCAT, traffic are initiated at $0.6 \mathrm{nmi}$ (within $D M O D_{\mathrm{wc}}=0.66 \mathrm{nmi}=4000 \mathrm{ft}$ ) at a bearing angle $\beta_{\text {тRAFFi }}$ in $\left[0^{\circ}\right.$, $360^{\circ}$ ) and move outer bound every $0.01 \mathrm{nmi}$ for each simulation run.

- For CAAT, traffic are initiated at $490 \mathrm{ft}\left(0.08 \mathrm{nmi}\right.$ within $\left.R_{\mathrm{NMAC}}=500 \mathrm{ft}\right)$ at a bearing angle $\beta_{\text {TrAfFi }}$ in $\left[0^{\circ}, 360^{\circ}\right)(\mathrm{e} . \mathrm{g}$. at $30^{\circ}$ in Fig. 10 and move outer bound every $10 \mathrm{ft}(0.0016 \mathrm{nmi})$ for each simulation run.

- The detected traffic bearing $\beta_{\text {TRAFFi }}$ and the traffic heading $\psi_{\text {TRAFFi }}$ are selected every $1^{\circ}$ in $\left[0^{\circ}, 360^{\circ}\right.$ ).

- Winds are introduced at $20 \mathrm{kt}$ in eight directions every $45^{\circ}$ in $\left[0^{\circ}, 360^{\circ}\right)$.

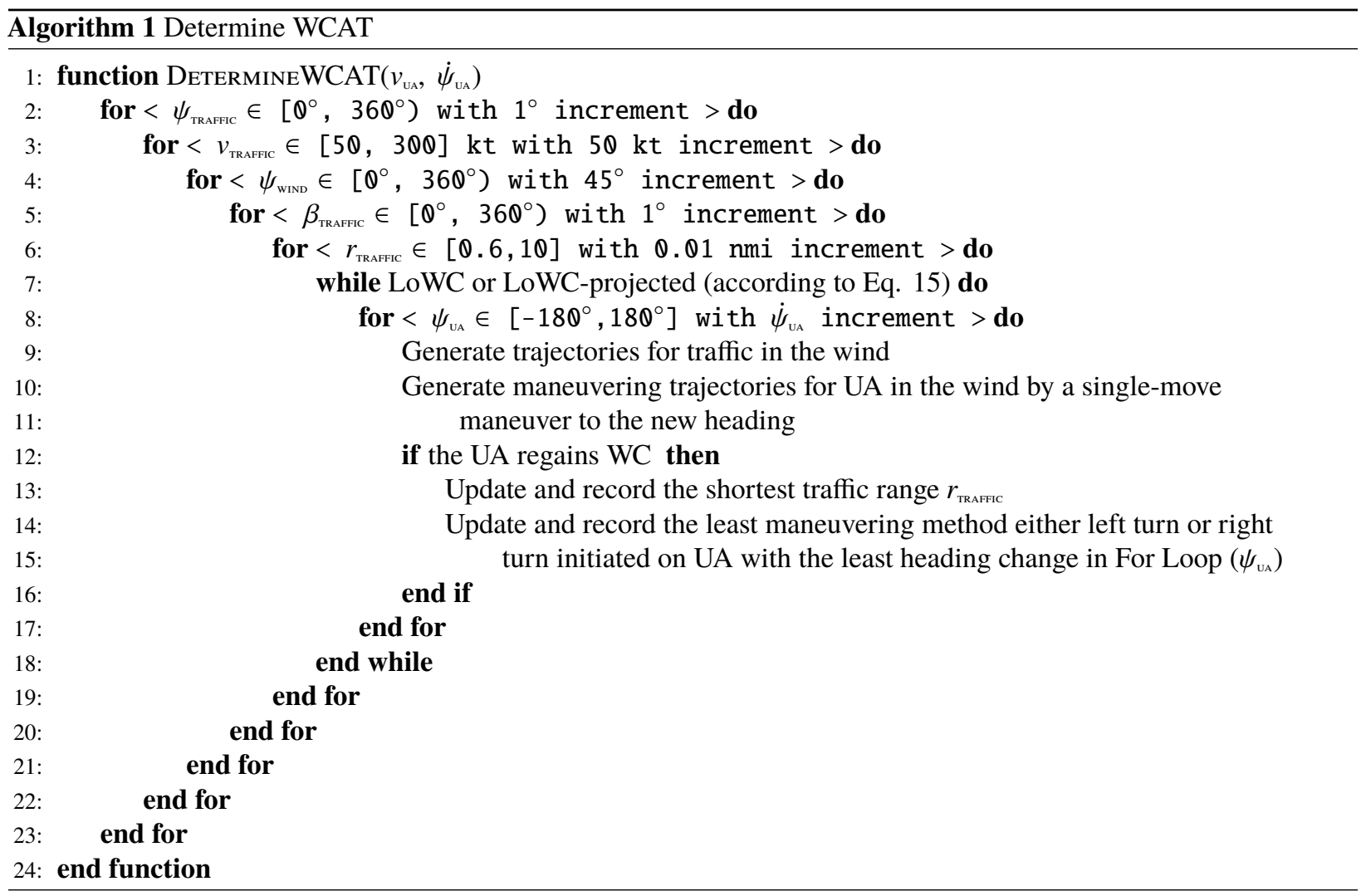

In addition, during $\mathrm{MC}$ simulations, for each simulated encounter, the closest traffic range and the least maneuvering method (left turn or right turn with the least heading change on the UA), as well as other related encounter parameters such as $v_{\mathrm{UA}}, \psi_{\mathrm{UA}}, v_{\mathrm{TRAFFI}}, \psi_{\mathrm{TRAFFIC}}, \beta_{\mathrm{TRAFFIC}}, v_{\mathrm{WIND}}, \psi_{\mathrm{WIND}}, H M D, V M D$, and $\tau_{\text {mod }}$, are saved in a SQLite local database [17] for further analysis. In this paper, they are first used to construct WCAT and CAAT lookup tables for real-time hazard identification in mid-air encounters. Later in the research, these encounter results will be used again as the encounter maneuvering guidance knowledge base to guide decision making in the mitigation solution module of the proposed DAA system in Fig. 2 


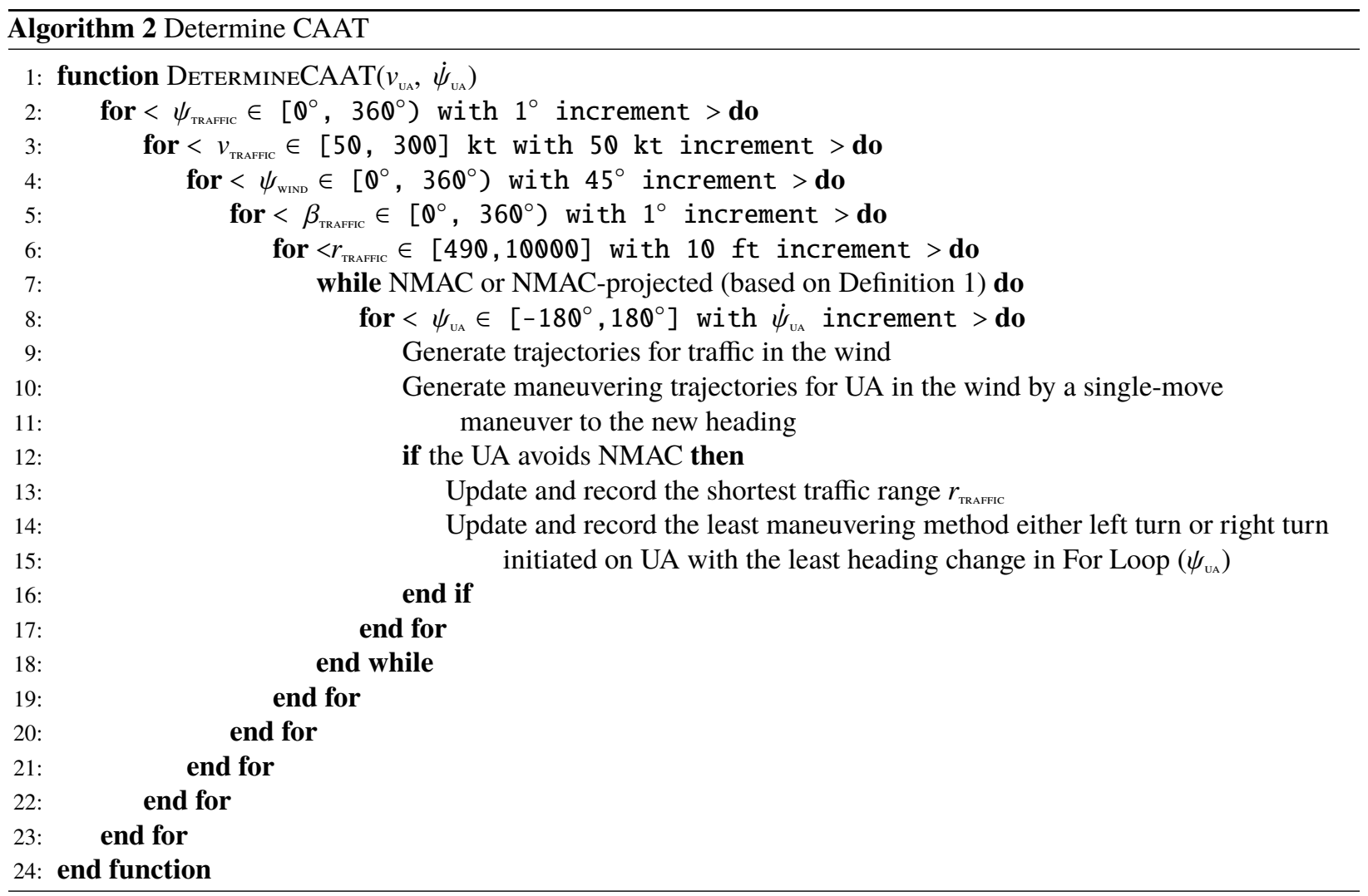

\section{Analysis and Results}

\section{A. Impact of WCAT and CAAT boundaries on wind uncertainties}

To analyze the impact of WCAT and CAAT boundaries on wind uncertainties (i.e., various wind speeds and wind directions), a $20 \mathrm{kt}$ gusty wind with a pattern [18] in Fig. 11] is introduced in MC simulations while locating WCAT and CAAT boundaries.

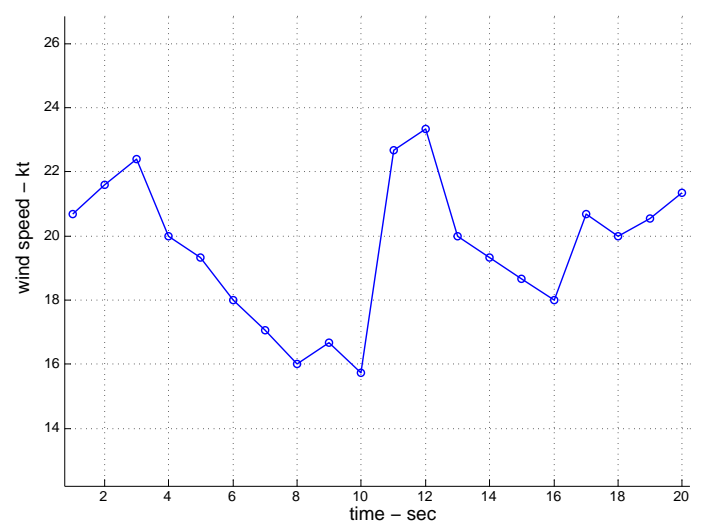

Fig. 11 An example of 20 -second gusty wind pattern with wind velocity jumps [18].

Followed by Algorithm 1 (Determine WCAT) and Algorithm 2(Determine CAAT) with the gusty winds introduced 
in eight wind directions from $0^{\circ}$ to $360^{\circ}$ every $45^{\circ}$, two $\mathrm{MC}$ simulations are performed in this section and encounter results are saved in the database as designated in Section III] Once these two MC simulations are completed, a data retrieving process is then performed to find the shortest horizontal range for UA to avoid LoWCs or NMACs at each bearing angle in $\left[0^{\circ}, 360^{\circ}\right)$ under one of the gusty wind conditions for nearby traffic operating in the heading angles from $0^{\circ}$ to $360^{\circ}$ every $1^{\circ}$ and the airspeeds from $50 \mathrm{kt}$ to $300 \mathrm{kt}$ every $50 \mathrm{kt}$.

For example, at the bearing angle $30^{\circ}$ for a $100 \mathrm{kt}$ operated traffic aircraft under a $20 \mathrm{kt}$ gusty wind in $45^{\circ}$ (southwest wind), 360 matched records are retrieved from the database for traffic in all heading angles from $0^{\circ}$ to $360^{\circ}$ every $1^{\circ}$, and then the alert threshold for this bearing angle $30^{\circ}$ is obtained by the shortest range among these 360 retrieved records. Similar to this example, data retrieving processes for all 360 bearing angles, six traffic airspeeds, and eight gusty wind directions are performed throughout the whole database and finally a number of WCAT and CAAT boundaries are obtained and plotted in this section.

\section{WCAT}

In Fig. 12, eight subfigures show small UA WCAT boundaries under 20 kt gusty winds in eight wind directions, where WCAT boundaries are rotated in various wind directions. For instance, WCAT boundaries aren the winds come from the left side of the UA (e.g., southwest wind in $45^{\circ}$ and northwest wind in $135^{\circ}$ ), and vice versa. Moreover, in each subfigure in Fig. 12, the size of WCAT boundaries are proportional to traffic velocities, namely, the faster the traffic velocities the bigger the WCAT boundaries. In addition, the UA front $\pm 120^{\circ}$ WCAT boundaries are generally larger than the UA rear ones due to faster relative velocities between UA and traffic.

During UAS operations, wind conditions around the UA usually vary over time and location, and no accurate wind velocity measurements are available for DAA sy — only estimates based on the airspeed and groundspeed of the UA are given. Thus, to overcome wind uncertainties on WCAT boundaries, we reorganize eight sets of WCAT boundaries in Fig. 12 into a set of wind-summarized WCAT boundaries in Fig. $13 \mathrm{~b}$ to cover encounters in various wind conditions. In general, the UA front WCAT boundaries in Fig. 13b are enlarged in comparison with the ones without wind in Fig. 13a In particular, the UA WCAT boundaries at the bearing angles $\left[95^{\circ}, 110^{\circ}\right]$ and $\left[250^{\circ}, 265^{\circ}\right]$ are enlarged dramatically due to the WCAT boundary rotations in various wind directions. For quantitative comparison between Fig. 13a and Fig. 13b we define a new variable, the threshold changing ratio (TCR)

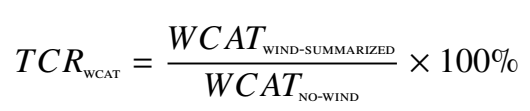

Two TCR diagrams of WCAT boundaries on Cartesia ordinates and on polar coordinates are plotted in Fig. 14 The TCRs of WCAT boundaries are increased by almost $25 \%$ between the wind-summarized WCAT and the no-wind WCAT at the bearing angles $\left[95^{\circ}, 110^{\circ}\right]$ and $\left[250^{\circ}, 265^{\circ}\right]$. In other words, these two traffic bearing regions are the most 


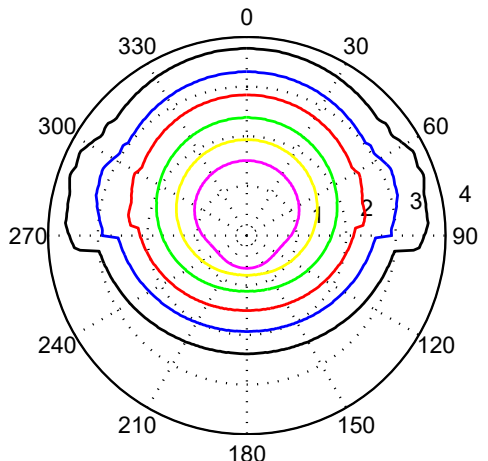

(a) $0^{\circ}$

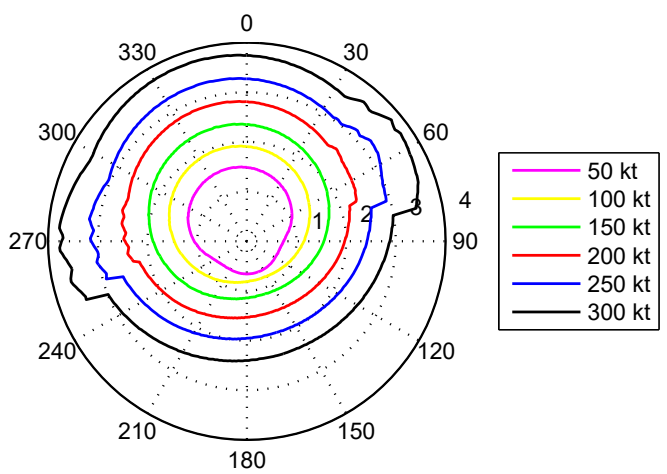

(c) $90^{\circ}$

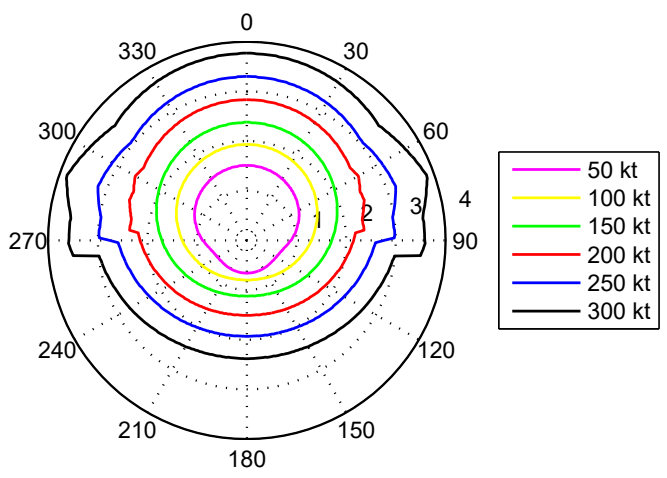

(e) $180^{\circ}$

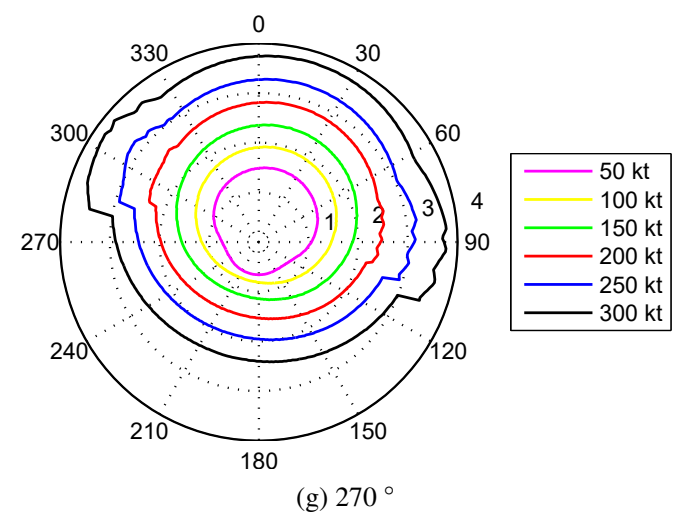

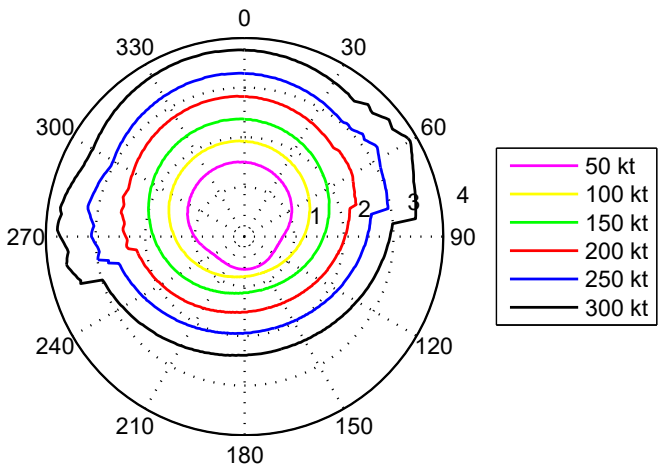

(b) $45^{\circ}$

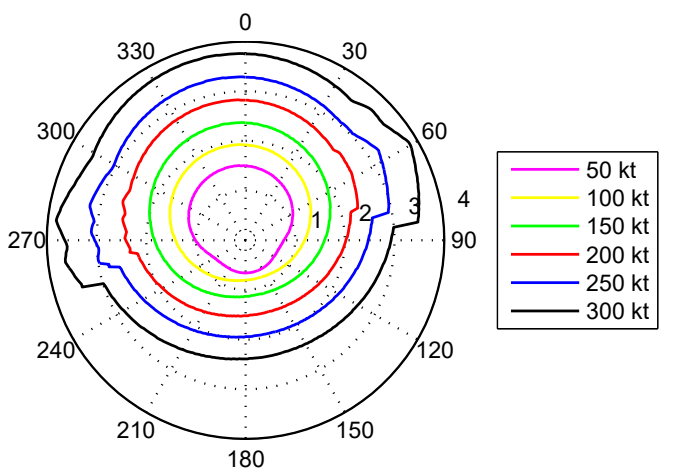

(d) $135^{\circ}$

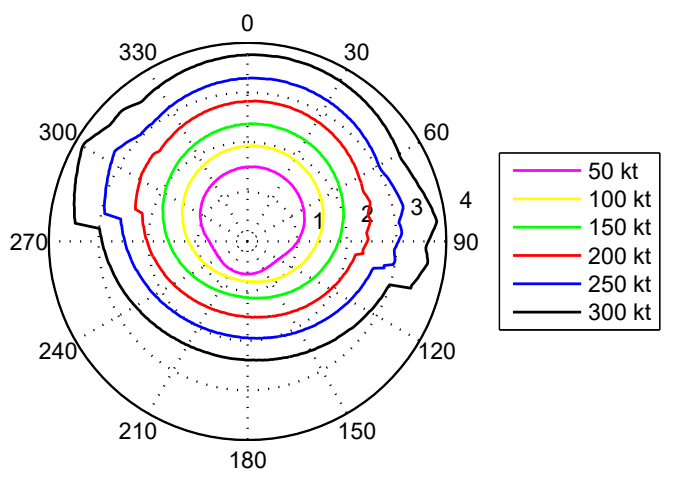

(f) $225^{\circ}$

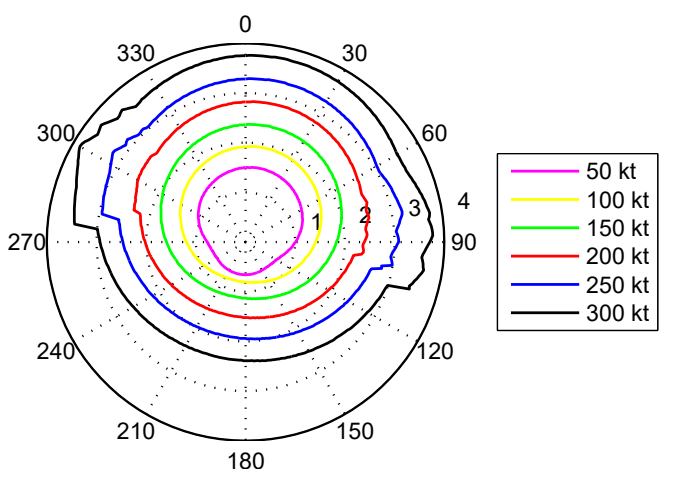

(h) $315^{\circ}$

Fig. 12 WCAT diagrams with $20 \mathrm{kt}$ gusty winds in eight directions (bearing in deg, range in nmi, UA at $75 \mathrm{kt}$, and traffic at various airspeeds). 


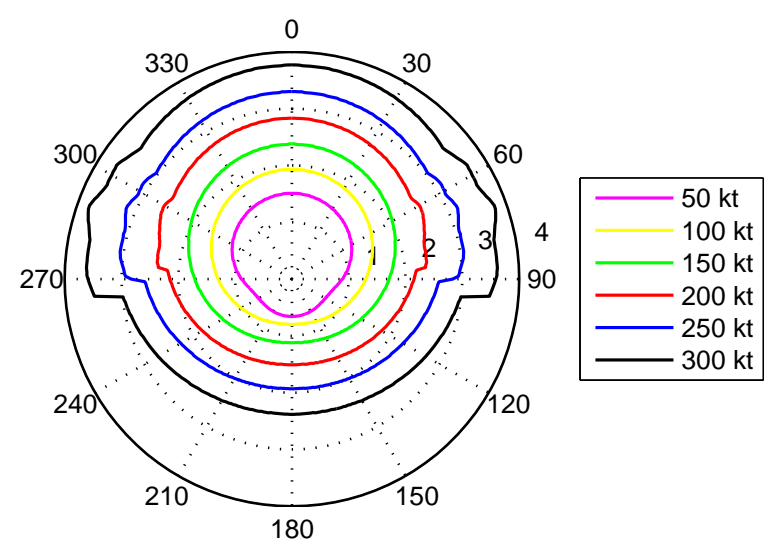

(a) WCAT obtained without wind.

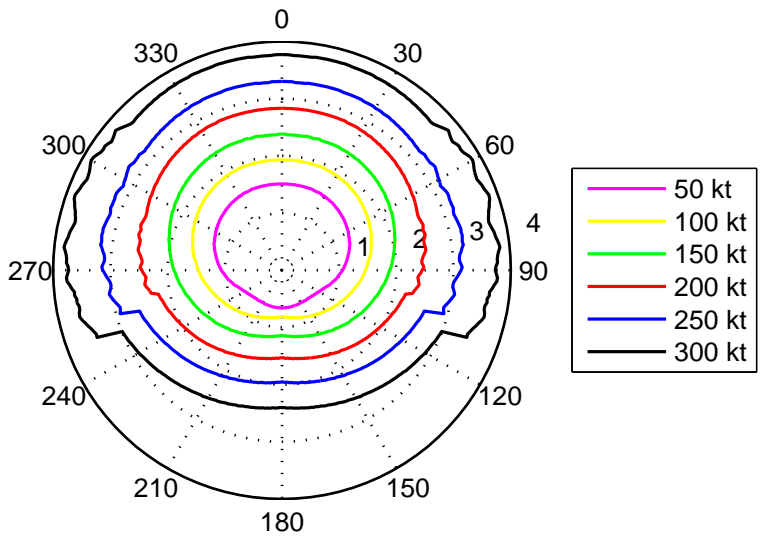

(b) Summarized with various $20 \mathrm{kt}$ gusty winds.

Fig. 13 Summarized WCAT diagrams (bearing in deg, range in nmi, UA at $75 \mathrm{kt}$ vs. traffic at various airspeeds).

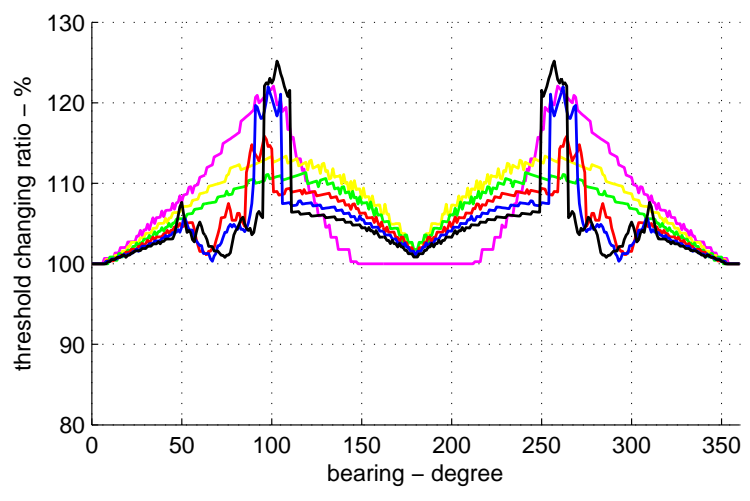

(a) On Cartesian coordinates.

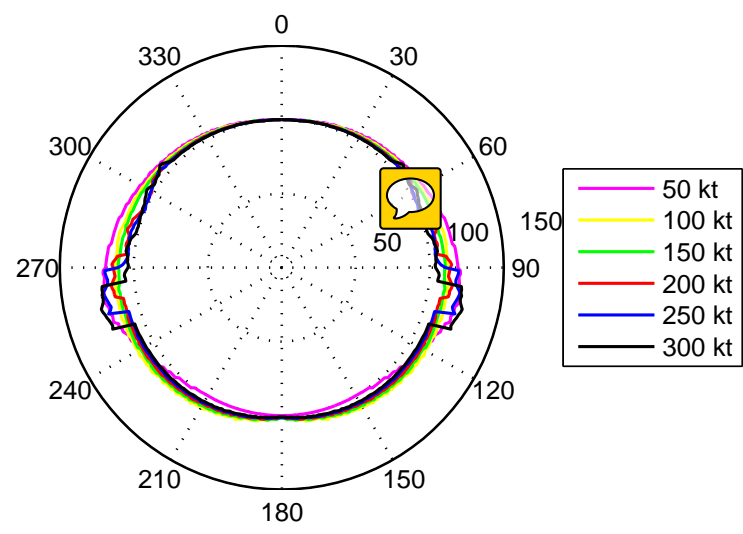

(b) On polar coordinates.

Fig. 14 Threshold changing ratio diagrams for the impact of WCAT boundaries on wind uncertainties (UA at $75 \mathrm{kt}$ and traffic at various airspeeds).

vulnerable regions for the PIC to identify hazards and initiate maneuvers to avoid LoWCs in various wind conditions during UAS operations.

\section{CAAT}

Eight subfigures in Fig. 15 show small UA CAAT boundaries under 20 kt gusty wind in eight wind directions, where CAAT boundaries are rotated into wind directions as those rotations occurred on WCAT boundaries. Moreover, in each subfigure in Fig. 15, the size of CAAT boundaries are proportional to traffic velocities, namely, the faster the traffic velocities the bigger the CDT boundaries.

Similar to the wind-summarized WCAT, we reorganize eight sets of CAAT boundaries in Fig. 15 into a set of wind-summarized CAAT boundaries in Fig. $16 \mathrm{~b}$ to cover encounters in various wind conditions. In general, the UA front CAAT boundaries in Fig. 16b are enlarged in comparison with the ones without wind in Fig. 16a. For quantitative 


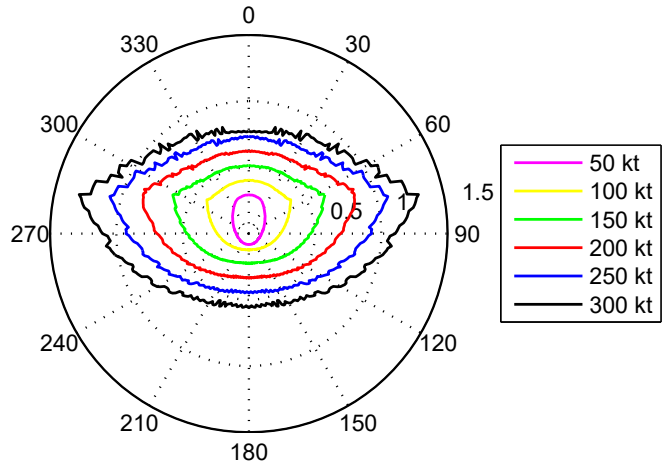

(a) $0^{\circ}$

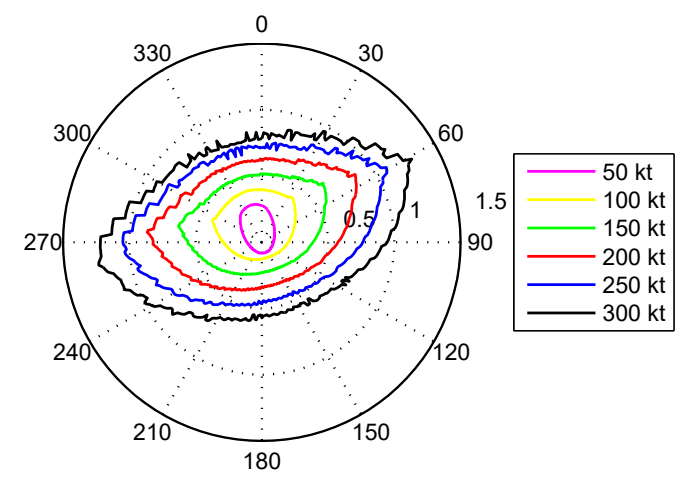

(c) $90^{\circ}$

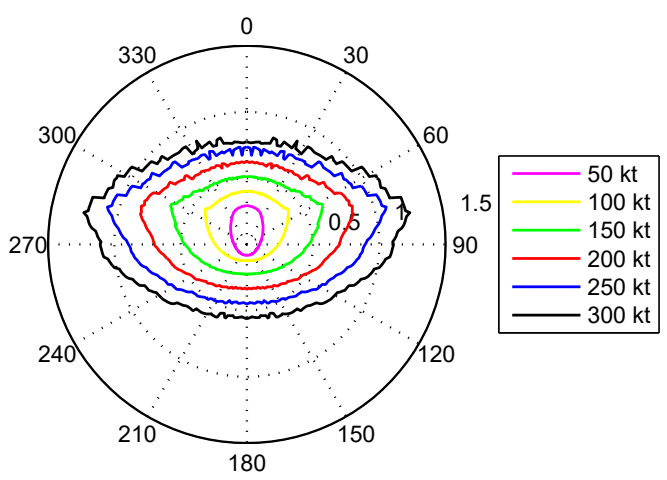

(e) $180^{\circ}$

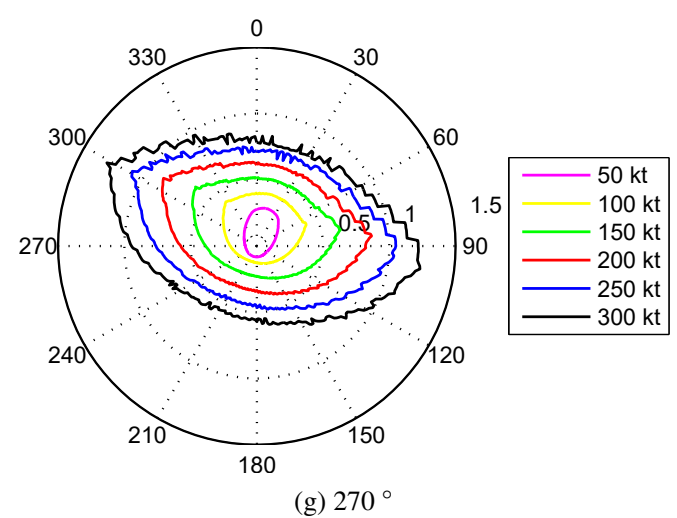

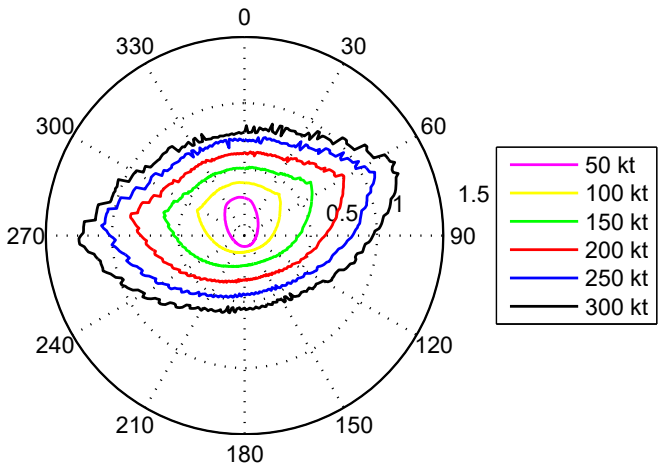

(b) $45^{\circ}$

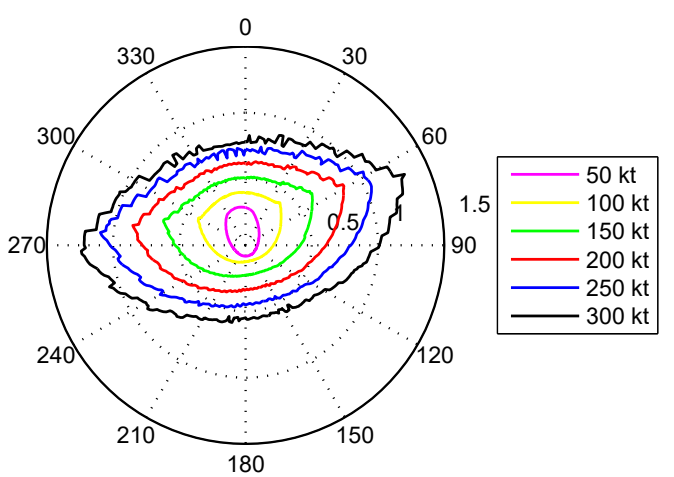

(d) $135^{\circ}$

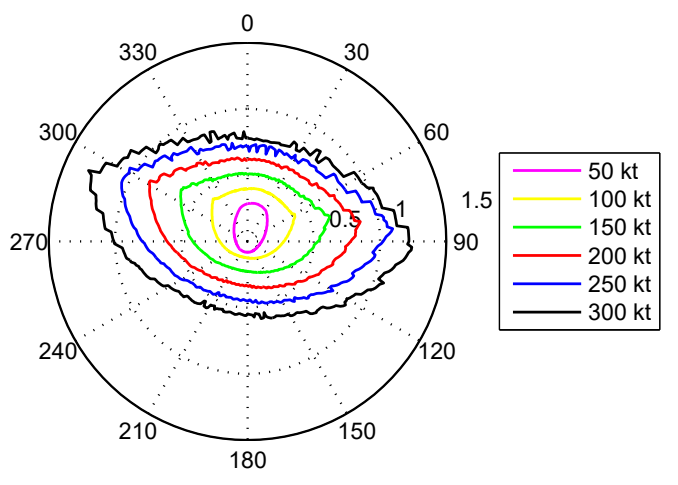

(f) $225^{\circ}$

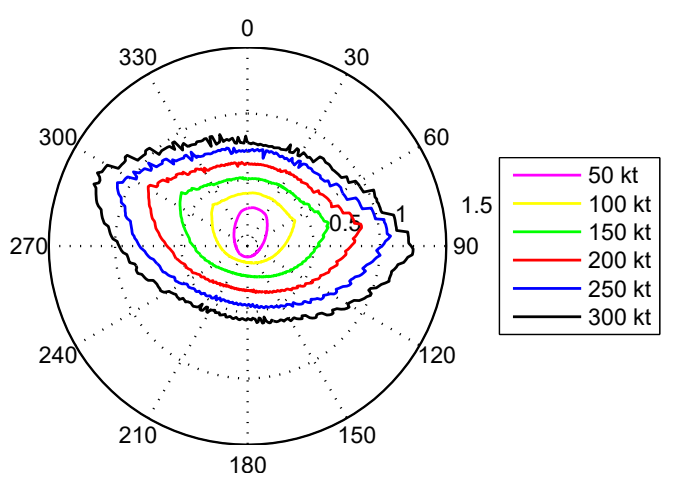

(h) $315^{\circ}$

Fig. 15 CAAT diagrams with $20 \mathrm{kt}$ gusty winds in eight directions (bearing in deg, range in nmi, UA at $75 \mathrm{kt}$, and traffic at various airspeeds). 


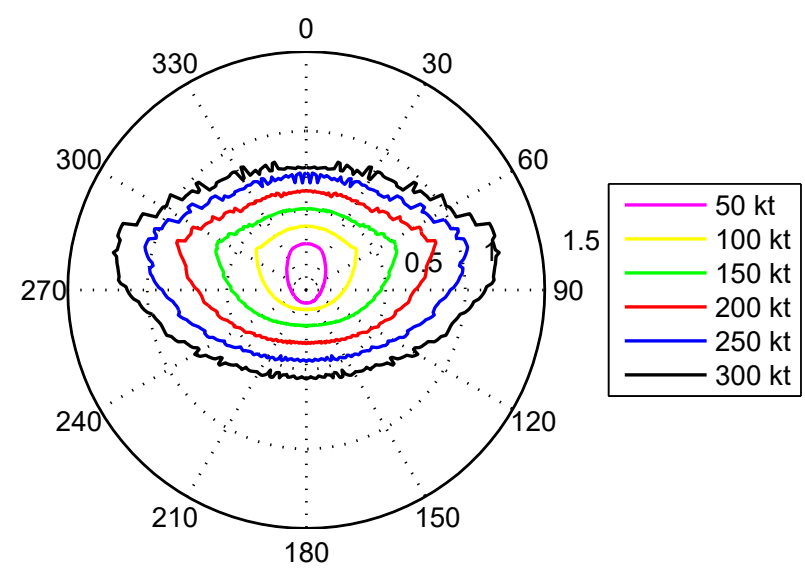

(a) CAAT obtained without wind.

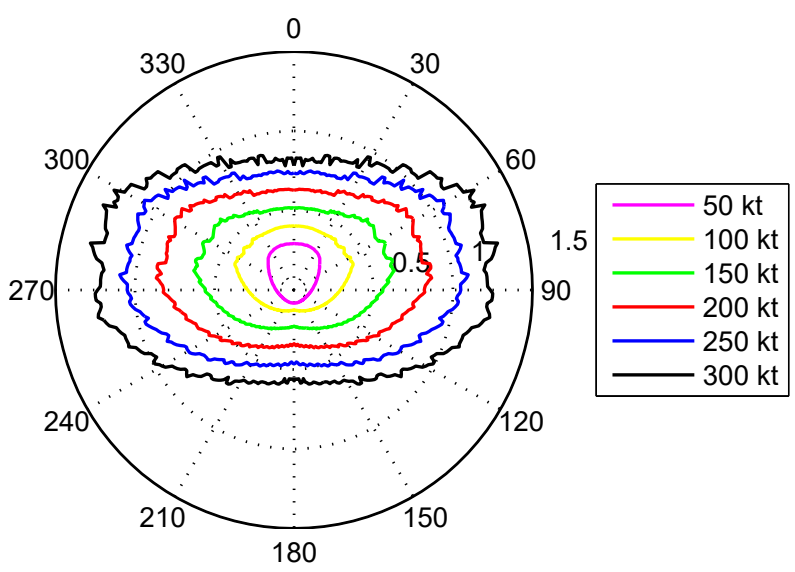

(b) Summarized with various 20 kt gusty winds.

Fig. 16 Summarized CAAT diagrams (bearing in deg, range in $\mathbf{n m i}$, UA at $75 \mathrm{kt}$, and traffic at various airspeeds).

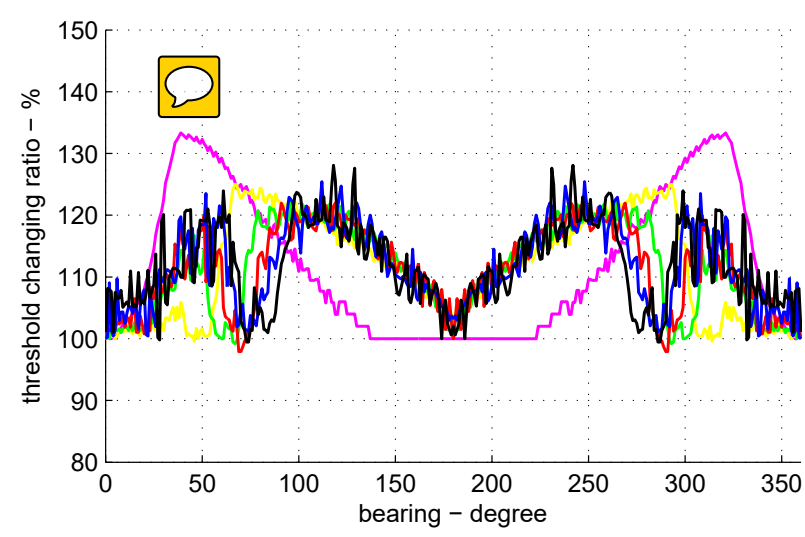

(a) On Cartesian coordinates.

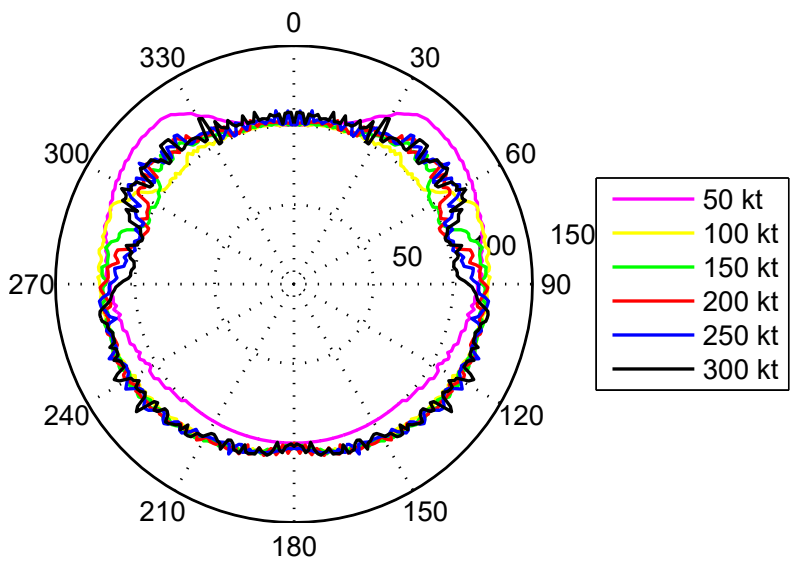

(b) On polar coordinates.

Fig. 17 Threshold changing ratio diagrams for the impact of CAAT boundaries on wind uncertainties (UA at $75 \mathrm{kt}$ and traffic at various airspeeds).

comparison between Fig. 16a and Fig. 16b, we define

$$
T C R_{\text {САAт }}=\frac{C A A T_{\text {wIND-SUмMARZZED }}}{C A A T_{\text {NO-WNND }}} \times 100 \%
$$

Two TCR diagrams of CAAT boundaries on Cartesian coordinates and on polar coordinates are plotted in Fig. 17 The TCRs of CAAT boundaries are about 10\% to 35\% increased between the wind-summarized CAAT and the no-wind CAAT in most bearing angles. Furthermore, Fig. 16a and Fig. 16b show that UA require longer horizontal ranges to avoid NMACs for traffic from the side regions of UA, such as at the bearing angles $\left[60^{\circ}, 120^{\circ}\right]$ and $\left[240^{\circ}, 300^{\circ}\right]$, because during UAS operations they are the most vulnerable bearing regions on CAAT boundaries in various wind conditions. 


\section{B. Real-time lookup tables}

Based on wind-summarized WCAT and CAAT boundaries in Fig. 13b and Fig. 16b, two lookup tables are constructed for real-time use. In the MC simulations, traffic at six airspeeds from $50 \mathrm{kt}$ to $300 \mathrm{kt}$ every $50 \mathrm{kt}$ are simulated so only six traffic airspeeds are available in the lookup tables. Moreover, in Fig. 13b and Fig. 16b, fast traffic corresponds with larger WCAT or CAAT boundaries of small UA. Therefore, a worst-case scenario method is designed to estimate WCAT or CAAT boundaries for small UA at each encounter during UAS operations. This worst-case scenario lookup table method can be summarized as follows:

1) Locate the airspeed range for traffic, and choose the upper bound as the airspeed for traffic in the lookup table search.

2) Use the rounded traffic detected bearing angle as the index to locate the WCAT or CAAT for small UA in lookup tables.

\section{Conclusion and Future Work}

In this paper, two MC simulation-based algorithms are designed and implemented to locate the WCAT and CAAT boundaries for small UAS, and the analytical results from MC simulations are used to form lookup tables to provide real-time WCAT and CAAT estimations in mid-air encounters, and help the PIC identify hazards and trigger avoidance maneuvers before a LoWC or NMAC occurs. Future work to locate more precise WCAT and CAAT boundaries for small UAS will require further MC simulations ceg more wind conditions (more wind speeds and wind directions), and more velocities for both UA and mid-air traffic.

\section{Acknowledgements}

The authors gratefully acknowledge Dilan Amarasinghe, Jon Stevenson, Bruno Miranda Artacho, and the RAVEN team for help and guidance in this research.

\section{Q \\ References}

[1] Federal Aviation Administration (FAA), "Sense and Avoid (SAA) for Unmanned Aircraft Systems (UAS)," Tech. rep., FAA Sponsored Sense and Avoid Workshop, 2009.

[2] Radio Technical Commission for Aeronautics (RTCA), "Minimum Operational Performance Standards (MOPS) for Detect and Avoid (DAA) Systems,” Document No: RTCA/DO-365, Washington, DC, RTCA, Inc., 2017.

[3] Fasano, G., Accado, D., Moccia, A., and Moroney, D., "Sense and avoid for unmanned aircraft systems," IEEE Aerospace and Electronic Systems Magazine, Vol. 31, No. 11, 2016, pp. 82-110. doi:10.1109/maes.2016.160116.

[4] International Civil Aviation Organization (ICAO), "Annex 2, to the Convention on International Civil Aviation, Rules of the 
Air, Chapter2 (par. 2.3.1),", 2005. URL https://wwW.icao.int/Meetings/anconf12/DocumentArchive/an02_cons\% 5B1\%5D.pdf.

[5] Federal Aviation Administration (FAA), "Code of Federal Regulations Title 14 Part 91 Subpart A Section 91.3 (14CFR91.3(a))," U.S. Government Publishing Office, 2011. URLhttps://wWW .gpo.gov/fdsys/pkg/CFR-2011-title14-vol2/pdf/CFR2011-title14-vol2-sec91-3.pdf

[6] Zeitlin, A., "Progress on Requirements and Standards for Sense \& Avoid," Tech. rep., The MITRE Corporation, 2010. URL https://www.mitre.org/sites/default/files/pdf/10_2799.pdf

[7] Transport Canada (TC), “Staff Instruction,” Document No: SI 623-001, May 2017. URL https://wWw.tc.gc.ca/eng/ civilaviation/standards/general-recavi-uav-4161.html.

[8] Fang, S. X., "Risk-based Supervisory Guidance for Detect and Avoid involving Small Unmanned Aircraft Systems," phdthesis, Memorial University, St. John's, NL, Canada, 2017.

[9] Johnson, M., Mueller, E. R., and Santiago, C., "Characteristics of a Well Clear Definition and Alerting Criteria for Encounters between UAS and Manned Aircraft in Class E Airspace,” Eleventh USA/Europe Air Traffic Management Research and Development Seminar (ATM2015), 2015. URL http://www . atmseminar .org/seminarContent/seminar11/papers/ 388-Johnson_0123150804-Final-Paper-5-6-15.pdf

[10] Cook, S. P., Brooks, D., Cole, R., Hackenberg, D., and Raska, V., "Defining Well Clear for Unmanned Aircraft Systems," AIAA Infotech @ Aerospace, AIAA SciTech Forum, American Institute of Aeronautics and Astronautics, 2015. doi: 10.2514/6.2015-0481, URL https://doi .org/10.2514/6.2015-0481

[11] Wikle, J. K., McLain, T. W., Beard, R. W., and Sahawneh, L. R., "Minimum Required Detection Range for Detect and Avoid of Unmanned Aircraft Systems," Journal of Aerospace Information Systems, Vol. 14, No. 7, 2017, pp. 351-372. doi:10.2514/1.I010479, URL/https://doi.org/10.2514/1.I010479

[12] Hull, D. G., Fundamentals of Airplane Flight Mechanics, Springer-Verlag Berlin Heidelberg, 2007. doi:10.1007/978-3-54046573-7.

[13] Lee, S. M., Park, C., Johnson, M. A., and Mueller, E. R., "Investigating Effects of Well Clear Definitions on UAS Sense-AndAvoid Operations in Enroute and Transition Airspace," 2013 Aviation Technology, Integration, and Operations Conference, AIAA AVIATION Forum, American Institute of Aeronautics and Astronautics, 2013. doi:10.2514/6.2013-4308, URL http://dx.doi.org/10.2514/6.2013-4308

[14] Radio Technical Commission for Aeronautics (RTCA), "Minimum Operational Performance Standards (MOPS) for Traffic Alert and Collision Avoidance System II (TCAS II) Hybrid Surveillance,” Document No: RTCA/DO-300, Washington, DC, RTCA, Inc., 2006. 
[15] Sunday, D., "Distance between 3D Lines \& Segments," From softSurfer, Oct. 2017. URL http://geomalgorithms . com/a07_distance.html

[16] McGovern, S. M., Cohen, S. B., Truong, M., and Fairley, G., "Kinematics-based model for stochastic simulation of aircraft operating in the national airspace system,” 2007 IEEE/AIAA 26th Digital Avionics Systems Conference, 2007, pp. 3.B.2-1-3.B.2-11. doi:10.1109/DASC.2007.4391876.

[17] HWACI, “About SQLite,” Hipp, Wyrick \& Company, Inc., Oct. 2017. URL https://www.sqlite.org/about.html.

[18] Bierbooms, W. A. A. M., “Constrained stochastic simulation of wind gusts for wind turbine design,” phdthesis, Delft University Wind Energy Research Institute, 2009. URL https://repository.tudelft.nl/islandora/object/uuid: f1d1751477c0-4ed1-88ff-c46a1006f66d?collection=research 